\title{
Anticoagulation for Atrial Fibrillation: Is This the End of Warfarin? Not Just Yet
}

\author{
Michael Mallouppas ${ }^{1}$ and Vassilios Vassiliou ${ }^{2}$ \\ ${ }^{1}$ Department of Medicine, Addenbrooke's Hospital, Cambridge University Health Partners, Cambridge CB2 0QQ, UK \\ ${ }^{2}$ Department of Cardiology, Royal Brompton Hospital, London and Imperial College London, Sydney Street, London SW3 6NP, UK
}

Correspondence should be addressed to Vassilios Vassiliou; vassiliou@doctors.org.uk

Received 30 April 2013; Accepted 23 June 2013

Academic Editor: Hironori Nakagami

Copyright (C) 2013 M. Mallouppas and V. Vassiliou. This is an open access article distributed under the Creative Commons Attribution License, which permits unrestricted use, distribution, and reproduction in any medium, provided the original work is properly cited.

\begin{abstract}
Atrial fibrillation (AF) is the most common cardiac arrhythmia. Its prevalence is known to increase with age and with an aging population AF is likely to become even more common. Although sometimes patients with AF remain asymptomatic, it is now recognized that AF is far from "benign" conferring a significant risk increase in morbidity and mortality. Restoration of sinus rhythm and rate-limiting medication help with symptoms; however, anticoagulation remains essential in reducing thromboembolic risk. The uptake of appropriate anticoagulation with vitamin $\mathrm{K}$ antagonists has increased significantly in the last few decades and this review will analyze whether the new oral anticoagulants might prove to be even more effective than existing vitamin K antagonists.
\end{abstract}

\section{Introduction}

Atrial fibrillation (AF) is the most common cardiac arrhythmia affecting $1-2 \%$ of the general population [1]. Its prevalence can increase up to $15 \%$ [2] in people older than 80 years of age, and furthermore in some patient groups such as those with a permanent pacemaker, AF might have an even greater prevalence $[3,4]$. The once considered "benign" arrhythmia is now recognized to increase mortality by twofold and the risk of stroke by fivefold leading to increased hospital admissions, reduced quality of life, impaired exercise capacity, tachycardia-induced cardiomyopathy, and heart failure [5]. Although restoration of sinus rhythm and rate-limiting medications are recognized to help with the symptoms associated with atrial fibrillation [6], appropriate anticoagulation is the major step in reducing stroke and thromboembolic risk [7].

\section{Anticoagulation in AF}

Warfarin, a vitamin $\mathrm{K}$ antagonist, is the most commonly utilized oral anticoagulant to reduce the risk of stroke and thromboembolism in patients with AF. It can lead to up to a $60 \%$ relative risk reduction of stroke and thromboembolic events compared to placebo and with an absolute risk reduction of $2.7 \%$ per year only 37 patients need to be anticoagulated to prevent one episode of stroke or thromboembolism [8]. It also compares favorably with aspirin [9] and dual therapy with aspirin plus clopidogrel [10] and is duly recommended in national and international guidelines (ESC, ACCP, AHA, NICE, SIGN [1, 11-14]). However, warfarin does have many side effects including unpredictable pharmacokinetics in response to food and drug interactions and therefore regular monitoring is required even in stable patients [15]. Therefore, new oral anticoagulants (NOACs) that have a predictable response and negate the need for regular blood monitoring offer an attractive alternative. Such NOACs have been manufactured and have been approved for use in patients in both North America and Europe and are being promoted as warfarin substitutes for anticoagulation in patients with nonvalvular AF.

\section{Thromboembolic Risk Stratification and Anticoagulation}

Although anticoagulation is very important in reducing thromboembolic risk in patients with $\mathrm{AF}$, it is also recognized 
TABLE 1: How the two most commonly used stroke risk scoring systems can be calculated: $\mathrm{CHADS}_{2}$ has a maximum of six points and $\mathrm{CHA}_{2} \mathrm{DS}_{2}$-VASc a maximum of nine points, where the higher the number of points scored equates to higher thromboembolic risk.

\begin{tabular}{|c|c|c|c|c|c|}
\hline $\mathrm{CHADS}_{2}$ & Condition & Points allocated & $\mathrm{CHA}_{2} \mathrm{DS}_{2}$-VASc & Condition & Points allocated \\
\hline $\mathrm{C}$ & $\begin{array}{l}\text { Congestive heart failure or } \\
\text { left ventricular (LV) } \\
\text { dysfunction with ejection } \\
\text { fraction }(\mathrm{EF}) \leq 40 \%\end{array}$ & 1 & $\mathrm{C}$ & $\begin{array}{l}\text { Congestive heart failure or } \\
\text { left ventricular }(\mathrm{LV}) \\
\text { dysfunction with ejection } \\
\text { fraction }(\mathrm{EF}) \leq 40 \%\end{array}$ & 1 \\
\hline $\mathrm{H}$ & Hypertension & 1 & $\mathrm{H}$ & Hypertension & 1 \\
\hline A & Age $\geq 75$ & 1 & $\mathrm{~A}_{2}$ & Age $\geq 75$ & 2 \\
\hline $\mathrm{D}$ & Diabetes & 1 & $\mathrm{D}$ & Diabetes & 1 \\
\hline \multirow[t]{4}{*}{$\mathrm{S}_{2}$} & $\begin{array}{l}\text { Transient ischemic attack } \\
\text { (TIA), stroke, or another } \\
\text { thromboembolism }\end{array}$ & 2 & $\mathrm{~S}_{2}$ & $\begin{array}{l}\text { Transient ischemic attack } \\
\text { (TIA), stroke, or another } \\
\text { thromboembolism }\end{array}$ & 2 \\
\hline & & & $\mathrm{V}$ & $\begin{array}{l}\text { Vascular disease (e.g., } \\
\text { peripheral artery disease, } \\
\text { myocardial infarction, } \\
\text { aortic plaque) }\end{array}$ & 1 \\
\hline & & & A & Age $65-74$ & 1 \\
\hline & & & Sc & Sex category that is, female & 1 \\
\hline
\end{tabular}

that not everyone needs to be anticoagulated as the benefits of anticoagulation might not outweigh the risks associated with anticoagulation, predominantly severe bleeding occurring at about $0.5-1 \%$ per patient-year. Therefore, scores that estimate thromboembolic risk have been devised, based on individual patient risk factors. The two most commonly used scores are the $\mathrm{CHADS}_{2}$ and $\mathrm{CHA}_{2} \mathrm{DS}_{2}$-VASc scores (Table 1).

Various countries have developed their own guidance for anticoagulation in AF, (ACCP [11], NICE [13], SIGN [14]); however, an up-to-date international guidance comes from the European Society of Cardiology (ESC) 2010 [1] and its 2012 focused update [16]. The ESC recommends that anticoagulation should be considered in everyone that has even moderate risk of thromboembolism as predicted by a $\mathrm{CHA}_{2} \mathrm{DS}_{2}$-VASc $\geq 2$ as the $2.2 \%$ annual risk of stroke or thromboembolism is considered significant and outweighs the risk associated with anticoagulation. For patients with $\mathrm{CHA}_{2} \mathrm{DS}_{2}-\mathrm{VASc}=1$, the ESC recommends that either anticoagulation or aspirin therapy is considered appropriate, with a comment that the former is preferable. Only the patients at very low risk with $\mathrm{CHA}_{2} \mathrm{DS}_{2}$-VASc $=0$ are advised to refrain from anticoagulation as this risk is smaller than the risk associated with anticoagulation. For these patients, the ESC recommends either aspirin or no therapy, with the latter being preferable.

The $\mathrm{CHA}_{2} \mathrm{DS}_{2}$-VASc, for example, attempts to estimate the individual risk score as demonstrated in Table 2.

\section{Warfarin and Novel Oral Anticoagulants}

Warfarin has many advantages, justifying its use as the major anticoagulant since its discovery in 1922 [17]. It is efficacious especially when in therapeutic range, inexpensive, and with once daily dosing allows for good adherence. In case where reversal is required, for example, in intracranial bleeding or nonelective surgery such as polytrauma, one could choose
TABLE 2: Using the $\mathrm{CHA}_{2} \mathrm{DS}_{2}$-VASc score, an annual adjusted risk score for stroke can be estimated, and the benefits of anticoagulation can be considered.

\begin{tabular}{lc}
\hline $\mathrm{CHA}_{2} \mathrm{DS}_{2}$-VASc score & Risk (annual adjusted risk) \\
\hline 0 & $0 \%$ \\
1 & $1.3 \%$ \\
2 & $2.2 \%$ \\
3 & $3.2 \%$ \\
4 & $4.0 \%$ \\
5 & $6.7 \%$ \\
6 & $9.8 \%$ \\
7 & $9.6 \%$ \\
8 & $6.7 \%$ \\
9 & $15.2 \%$ \\
\hline
\end{tabular}

from a plethora of agents depending on how quickly reversal is required ranging from vitamin $\mathrm{K}$ (for slow reversal) to prothrombin complex and fresh frozen plasma for urgent reversal. Blood testing has been considered traditionally a disadvantage, and although admittedly inconvenient to patients, it does have the hidden advantage that it allows physicians to monitor adherence, something particularly useful, for example, before elective electrical direct current cardioversion.

Because warfarin has unpredictable pharmacokinetics, it does mean that blood testing is required frequently even in stable patients [15]. Warfarin can be affected by different pharmacogenetics like the cytochrome P450 2C9 gene and vitamin $\mathrm{K}$ epoxide reductase complex 1 gene as well as various substances that act as cytochrome P450 inhibitors or inducers like other medications, food, and alcohol, and this instability in anticoagulation can increase the risk of hemorrhagic complications and stroke. This unpredictability of response and requirement for life-long monitoring in particular provides an area where NOACs might excel over 
warfarin both in terms of convenience and efficacy. Currently there are 3 NOACs licensed for anticoagulation in AF by the Food and Drug Administration (FDA) in the USA and the European Medicine Agency (EMA) in Europe. These are dabigatran etexilate, rivaroxaban, and apixaban and will be discussed in this review.

\section{Dabigatran Etexilate}

Dabigatran etexilate was the first NOAC to be licensed by both FDA and EMA following the publication of the RE-LY trial [18]. Etexilate is a prodrug that is rapidly converted to dabigatran, a direct, competitive inhibitor of thrombin with bioavailability of $6.5 \%$ and $80 \%$ renal excretion. Although it needs to be taken twice daily, its main advantage is that it does not require blood monitoring. In the RE-LY trial, 18,113 patients with non-valvular $\mathrm{AF}$ and one risk factor for stroke/thromboembolism were equally randomized in three groups: group 1 received warfarin (target INR of 2.03.0), group 2 received dabigatran $150 \mathrm{mg}$ twice daily, and group 3 received dabigatran $110 \mathrm{mg}$ twice daily. Stroke or systemic embolism occurred in 199 patients on warfarin (1.69\% per annum), 182 patients on dabigatran $110 \mathrm{mg}(1.53 \%$ per annum, hazard ratio $0.91,95 \% \mathrm{CI}=0.74-1.11 ; P<0.001$ for inferiority), and 134 patients on the $150 \mathrm{mg}$ twice daily dabigatran dose (1.11\% per annum, hazard ratio $0.67,95 \%$ CI $=0.53-0.82 ; P<0.001$ for superiority).

Thus, both doses of dabigatran proved noninferior to warfarin with the higher dose also being superior to warfarin in reducing stroke and systemic embolism. The higher dose dabigatran was, however, associated with higher gastrointestinal bleeding when compared with warfarin something not seen with the lower dose dabigatran.

There was also a concern that the $150 \mathrm{mg}$ dose of dabigatran was associated with a higher number of myocardial infarctions $(0.74 \%$ versus $0.53 \%, P=0.048)$ when the paper was initially published. Subsequently, the authors published further retrospective data acquired following the termination of the trial suggesting that this was not correct and dabigatran was not associated with increased myocardial infarctions [19]. However, the fact that dabigatran could lead to increased myocardial infarctions should not be dismissed in view of the retrospective nature of the data provided by the authors and furthermore, according to a recent meta-analysis dabigatran increased the relative risk of myocardial infarcts by 33\% compared to warfarin, enoxaparin, or placebo [20].

One important aspect that needs to be appreciated, however, is that overall mortality was similar in all groups

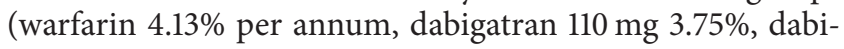
gatran $150 \mathrm{mg} 3.64 \%$ ) despite the reduced rates of stroke and bleeding observed with the higher and lower dose of dabigatran, respectively. In an attempt to explain this, Harper and colleagues [21] reviewed patients with bleeding on dabigatran and concluded that amongst other risk factors (renal function, age, and body weight) the lack of a reversing agent was a contributory factor that increased mortality and therefore this should be discussed with all patients prior to initiation of dabigatran.

\section{Rivaroxaban}

Rivaroxaban was the first oral direct factor Xa inhibitor both to complete a Phase III trial, the ROCKET-AF trial [22], but also to gain approval both from FDA and EMA. With a bioavailability of over $80 \%$ and with $65 \%$ of it renally excreted, it is the only NOAC approved to date that allows a once-daily regime.

ROCKET-AF was a randomized, double-blinded and double-dummy study of 14,264 patients with AF and a $\mathrm{CHADS}_{2} \geq 2$. Patients received either rivaroxaban $20 \mathrm{mg}$ once daily ( $15 \mathrm{mg}$ if creatinine clearance was $30-49 \mathrm{~mL} / \mathrm{min}$ ) or dose adjusted warfarin to maintain INR 2.0-3.0.

In the intention-to-treat analysis, stroke or systemic embolism occurred in 269 patients on rivaroxaban (2.1\% per annum, $P=0.0001$ for inferiority, $P=0.12$ for superiority) and 306 patients on warfarin (2.4\% per annum).

Major bleeding events were similar with both groups (3.6\% per annum with rivaroxaban, 3.4\% with warfarin). Intracranial bleeding was, however, significantly reduced with rivaroxaban $(0.5 \%$ versus $0.7 \%$ per annum, hazard ratio $0.67,95 \%$ CI $0.47-0.93, P=0.02)$. This was counterbalanced by an increased rate of major gastrointestinal bleeding with rivaroxaban (3.2\% versus $2.2 \%$ per annum, $P<0.001$ ).

As with dabigatran, no benefit was seen in overall mortality. One major criticism for rivaroxaban, however, was that the time spent in therapeutic range for patients on warfarin was only $55 \%$. This might be considered lower than what can be achieved in real life registries [23-25] and therefore could have potentially obscured any advantages in favor of warfarin.

\section{Apixaban}

Apixaban was the second factor Xa inhibitor that received approval both by FDA and EMA following publication of the ARISTOTLE trial [26], a randomized, double-blind, doubledummy, and noninferiority trial of 18,201 patients with nonvalvular AF and at least one risk factor for thromboembolism. Unlike rivaroxaban its half-life is 12 hours thus requiring twice daily dosage and it is $25 \%$ renally excreted making it therefore potentially safer in patients with mild renal failure when compared to dabigatran (Table 3). Patients were randomized equally to receive either warfarin aiming for INR 2.0-3.0 or apixaban $5 \mathrm{mg}$ twice daily $(2.5 \mathrm{mg}$ if two or more of the following existed: age $>80$, weight $<60 \mathrm{~kg}$, or creatinine $>133 \mu \mathrm{mol} /$ liter). Stroke or systemic embolism occurred in 212 patients taking apixaban and 265 patients taking warfarin ( $1.27 \%$ versus $1.60 \%$ per annum, hazard ratio $0.79,95 \%$ CI $0.66-0.95, P<0.001$ for inferiority, $P=0.01$ for superiority). Hemorrhagic stroke was reduced by $49 \%(P<0.001)$ and ischemic/undefined stroke by $8 \%(P=0.42)$ with apixaban. Fatal or disabling stroke occurred in 117 patients on warfarin (0.71\% per annum) and 84 patients on apixaban $(0.5 \%$ per annum). Mortality from any cause occurred in $3.52 \%$ per annum with apixaban compared with $3.94 \%$ per annum with warfarin (hazard ratio $0.89,95 \%$ CI $0.8-0.99, P=0.047$ ) thus making apixaban the only one of the three NOACs to show a benefit over warfarin in mortality. However, death from cardiovascular causes (including hemorrhagic stroke) 
TABLE 3: Showing the relative pharmacokinetics of the novel oral anticoagulants.

\begin{tabular}{lccc}
\hline & Dabigatran & Rivaroxaban & Apixaban \\
\hline Target & Thrombin & Factor Xa & Factor Xa \\
Dose & Twice daily & Once daily & Twice daily \\
Half-life in hours & $12-17$ & $5-9$ (but effect last for 24 hours) & 12 \\
Renal excretion & $80 \%$ & $25 \%$ & $33 \%$ \\
Hepatic excretion & $20 \%$ & $75 \%$ & $67 \%$ \\
\hline
\end{tabular}

and death from noncardiovascular causes (including fatal bleeding other than hemorrhagic stroke) was not significantly different between apixaban and warfarin.

Apixaban was also criticized that the time in therapeutic range for patients on warfarin during the ARISTOTLE trial was only $62 \%$ [27] again raising the possibility that this underestimated the benefit of well-controlled warfarin therapy.

Apixaban has also been the only NOAC to be compared to aspirin for stroke prophylaxis in patients who were intolerant of warfarin in the AVERROES trial [28]. Stroke or systemic embolism occurred in 51 patients on apixaban and 113 patients on aspirin (1.6\% versus $3.7 \%$ per annum, hazard ratio = 0.45 , 95\% CI $0.32-0.62, P<0.001)$. Mortality was nonsignificantly reduced with apixaban compared to aspirin (3.5\% per annum versus $4.4 \%$ per annum, hazard ratio 0.79 , 95\% CI $0.62-1.02, P=0.07)$. This trial thus confirmed that for patients who were intolerant of warfarin (or indeed refused warfarin), apixaban was a safer alternative than aspirin.

Furthermore, apixaban has also been to date the only NOAC used to compare anticoagulation with warfarin for patients in AF categorized according to two thromboembolic risk scores $\left(\mathrm{CHADS}_{2}\right.$ and $\mathrm{CHA}_{2} \mathrm{DS}_{2}$ VASc) and one bleeding score (HAS-BLED) [29]. In a subanalysis of patient risk in the ARISTOTLE trial, the authors detected no statistical heterogeneity of response across the different risk categories concluding that the most likely explanation for this was that apixaban benefited all patients, independently of thromboembolic and bleeding risk. Alternatively, as stated in an accompanying editorial [30], given that this subanalysis of the ARISTOTLE trial was not sufficiently powered to confirm such a difference, caution was advised in the interpretation of the results. Careful analysis of the results suggested that the numbers needed to treat (NNT) to prevent one stroke varied significantly from approximately 120 for those with $\mathrm{CHADS}_{2}$ $=1$ and around 60 for those with $\mathrm{CHADS}_{2} \geq 3$, indicating that patients with a higher thromboembolic risk might benefit more from a NOAC and this could significantly affect cost effectiveness.

\section{Should Patients with High Bleeding Risk Be Anticoagulated?}

A recent analysis from Denmark assessed patients in AF on warfarin with both the $\mathrm{CHADS}_{2}$ and $\mathrm{CHA}_{2} \mathrm{DS}_{2}$-VASc scoring systems and the risk of bleeding using the HAS-BLED system. The findings support the view that anticoagulation should be offered even in patients at the highest risk of bleeding (HASBLED $\geq 3$ ), as paradoxically this is the group of patients who benefit the most (probably because they also have a significant thromboembolic risk) [31]. The same appears to be true for apixaban use in patients with AF [29] where the incidence of major bleeding was $3.46 \%$ for patients with HAS-BLED $\geq$ 3 and $2.25 \%$ for HAS-BLED $=2$ while intracranial bleeding was $0.23 \%$ and $0.38 \%$, respectively. Based on the currently available evidence the decision to anticoagulate an individual patient with AF remains a nuanced one, balancing the Scylla of thromboembolism against the Charybdis of hemorrhage, both risks imperfectly predicted by even the best currently available risk stratification scores. However, even if there is a high bleeding risk, following appropriate discussion with the patient, suitable candidates should be offered anticoagulation. Novel oral anticoagulants, particularly apixaban, might prove to be very cost effective in this high-risk subgroup of patients.

At present, therefore, the most important reason for estimating the bleeding risk is not to prevent initiation of anticoagulation but to identify patients who might benefit from risk factor modification, for example, stopping aspirin if not required, introducing antihypertensive medication and support to reduce alcohol intake in order to minimize bleeding risk [32].

\section{Current Guidelines and New Anticoagulants}

Most of the international guidelines have now issued updates to incorporate these new data in the management of AF. The ESC in a detailed recent update now recommends the novel anticoagulants as "broadly preferable to Vitamin K antagonists in the vast majority of patients with non-valvular AF" [16] though caution in strict adherence to current indications is advised due to limited experience in their use. The ACCP too now suggests dabigatran as the preferred oral anticoagulant in non-valvular AF [11]. Therefore, is it the time to offer a NOAC to every patient with AF? This is probably not the case, at least not yet, as demonstrated in the following scenarios.

9.1. Case 1. An 82-year-old man with hypertension and AF who has been on warfarin for six years. He is stable with an INR in therapeutic range $>80 \%$ of the time. He requires blood monitoring every 6 weeks and is happy with the necessary dietary restrictions. He is seen in clinic and asks whether he should be taking a NOAC.

This is a common scenario, seen in most cardiology outpatient clinics. It is important to emphasize to the patient (and any relatives) that none of the main trials compared a NOAC with very good warfarin control. There is simply 
no evidence to support that switching to a NOAC will reduce either his risk of bleeding or thromboembolism. The advice should be to continue with warfarin. Had his time in therapeutic range been $<60 \%$ instead, then a NOAC could have been offered.

9.2. Case 2. A 35-year-old man with no risk factors for thromboembolism is scheduled to undergo electrical cardioversion for isolated AF.

Warfarin monitoring offers the unintentional benefit of adherence monitoring. Patients prior to electrical cardioversion need to be appropriately anticoagulated for a period of a few weeks prior to the procedure and documented INR within therapeutic range confirm this. This is not the case for any of the NOACs and therefore if patients miss any dose (either intentionally or unintentionally), then cardioversion would increase the risk of stroke. Patients should be treated with warfarin before the cardioversion and a few weeks after the cardioversion and then their anticoagulation can be reassessed and at this stage a NOAC might be appropriate.

9.3. Case 3. An 86-year-old woman with AF with previous TIA, hypertension, and peripheral vascular disease who lives alone in an area with no easy access to a clinic or community practitioner to monitor INR. Her time in therapeutic range for the last year was 50\%. Two months ago she had a significant bruising after a fall and her INR was found to be 5.4. She refused to take warfarin since then.

A patient who spends significant time outside therapeutic range is at risk both of significant bleeding when the INR is high and ischemic stroke when the INR is low. This woman has a $\mathrm{CHA}_{2} \mathrm{DS}_{2}-\mathrm{VASc}=7$. Her annual predicted risk of thromboembolism is $10 \%$ and therefore she would benefit greatly from anticoagulation. Given the difficulties in maintaining warfarin within therapeutic range, it appears that the risks of warfarin outweigh the potential benefits. Antiplatelet therapy either with aspirin monotherapy or dual therapy with aspirin and clopidogrel will only reduce this patient's thromboembolic risk moderately. Therefore, in keeping with the findings of the AVERROES trial [28], apixaban $2.5 \mathrm{mg}$ twice daily would be the most appropriate NOAC to use.

9.4. Case 4. A 55-year-old gentleman with permanent AF, diabetes, hypertension had a coronary drug-eluding stent in his right coronary artery 6 months ago. He tolerated triple therapy (aspirin, warfarin, and clopidogrel) for 6 months and the interventional cardiologists were happy to continue with clopidogrel and warfarin. His time in therapeutic INR is 60$70 \%$ and is asking whether warfarin could be substituted by one of the NOACs.

With an aging population facing both coronary disease and $\mathrm{AF}$, this scenario will become more common. A NOAC will not offer any protection from stent thrombosis. Clear guidance from Europe [1] and US [11] recommends triple anticoagulation for 6 months at least. From there on warfarin and clopidogrel are appropriate for another 6 months. The use of a NOAC + clopidogrel (or NOAC and aspirin) might prove superior to warfarin and clopidogrel but this has not been assessed in a trial. There have been some concerns about dabigatran and an increased risk of MI. In this case, on the basis of current evidence, it is appropriate to continue warfarin and clopidogrel and in the future the patient might even continue on warfarin monotherapy [1]. If, however, he refuses warfarin, it would be acceptable to consider a combination of clopidogrel and apixaban or clopidogrel and rivaroxaban although there is no evidence to support this at present.

9.5. Case 5. A 72-year-old man with hypertension, diabetes, and asymptomatic permanent AF refuses to consider warfarin and has been on aspirin monotherapy for seven years.

The results of the AVERROES study [28] indicate that this patient (who refused to consider anticoagulation) will have prognostic benefit if he takes apixaban $5 \mathrm{mg}$ twice daily. Although not directly compared with aspirin, the other two NOACs, dabigatran in the RE-LY trial [18] and rivaroxaban in the ROCKET-AF trial [22], are also likely to benefit this patient. The most cost-effective NOAC should therefore be considered for this patient. If, however, cost effectiveness is similar across the three options, then apixaban is the one that has the most evidence to support its use in this case.

It is important to highlight that NOACs can only be used in patients with isolated non-valvular AF. They are not appropriate for patients with AF and other co-existing pathology, for example, a metallic mitral valve. Although a NOAC should not be offered routinely as a first-line treatment for anticoagulation in isolated $\mathrm{AF}$, they can provide an excellent alternative to warfarin, particularly for patients with a low percentage time in therapeutic range (Case 3). Physicians should therefore monitor time spent in therapeutic range during clinic appointments as now there is a possibility of switching warfarin to a NOAC that is likely to be more effective for such patients.

\section{Conclusion}

The NOACs increase the therapeutic armamentarium available for patients with non-valvular AF who require thromboembolic prophylaxis and are likely to shape the future of anticoagulation. However, on the basis of the evidence currently available, considering the benefits, risks, and convenience against their cost effectiveness in "real life" clinical practice [33], one cannot recommend any of the NOACs as panacea and first-line therapy for everyone. Many patients, particularly those with high thromboembolic and bleeding risk, with poor INR control or who refuse warfarin therapy, are likely to benefit more from a NOAC. Over the next few years, these groups of patients should be offered a NOAC initially. Warfarin is likely to remain in use for the majority of patients with AF for the next few years and is likely to celebrate its centurial birthday. However, with the current generation of NOACs and the likelihood of new improved ones that will follow over the next decade, warfarin will probably lose its place as the most commonly used anticoagulant for $\mathrm{AF}$. 


\section{References}

[1] A. J. Camm, P. Kirchhof, G. Y. Lip et al., "Guidelines for the management of atrial fibrillation: the task force for the management of atrial fibrillation of the European Society of Cardiology (ESC)," European Heart Journal, vol. 31, no. 19, pp. 2369-2429, 2010.

[2] J. Heeringa, D. A. M. Van Der Kuip, A. Hofman et al., "Prevalence, incidence and lifetime risk of atrial fibrillation: the Rotterdam study," European Heart Journal, vol. 27, no. 8, pp. 949-953, 2006.

[3] V. S. Vassiliou, A. Farag, E. McIntosh, and I. Williams, "Pacemaker clinics and anticoagulation for atrial fibrillation," Europace, vol. 14, no. 9, p. 1375, 2012.

[4] S. Cabrera, J. Mercé, R. De Castro et al., "Pacemaker clinic: an opportunity to detect silent atrial fibrillation and improve antithrombotic treatment," Europace, vol. 13, no. 11, pp. 15741579, 2011.

[5] J. R. Ehrlich and S. H. Hohnloser, "Milestones in the management of atrial fibrillation," Heart Rhythm, vol. 6, no. 11, supplement, pp. S62-S67, 2009.

[6] C. Stellbrink, "Arrhythmia recurrence after ablation of atrial fibrillation: should we be concerned about sleep apnoea?" Europace, vol. 12, no. 8, pp. 1051-1052, 2010.

[7] M.-J. Cha, Y. D. Kim, H. S. Nam, J. Kim, D. H. Lee, and J. H. Heo, "Stroke mechanism in patients with non-valvular atrial fibrillation according to the CHADS2 and CHA2DS2-VASc scores," European Journal of Neurology, vol. 19, no. 3, pp. 473479, 2012.

[8] R. G. Hart, L. A. Pearce, and M. I. Aguilar, "Meta-analysis: antithrombotic therapy to prevent stroke in patients who have nonvalvular atrial fibrillation," Annals of Internal Medicine, vol. 146, no. 12, pp. 857-867, 2007.

[9] H. Sato, K. Ishikawa, A. Kitabatake et al., "Low-dose aspirin for prevention of stroke in low-risk patients with atrial fibrillation: Japan atrial fibrillation stroke trial," Stroke, vol. 37, no. 2, pp. 447451, 2006.

[10] S. Connolly, J. Pogue, R. Hart et al., "Clopidogrel plus aspirin versus oral anticoagulation for atrial fibrillation in the Atrial fibrillation Clopidogrel Trial with Irbesartan for prevention of Vascular Events (ACTIVE W): a randomized controlled trial," The Lancet, vol. 367, no. 9526, pp. 1903-1912, 2006.

[11] G. H. Guyatt, E. A. Akl, M. Crowther, D. D. Gutterman, and H. J. Schünemann, "Executive summary: antithrombotic therapy and prevention of thrombosis, 9th ed: American College of Chest Physicians evidence-based clinical practice guidelines," Chest, vol. 141, no. 2, supplement, pp. 7S-47S, 2012.

[12] K. L. Furie, L. B. Goldstein, G. W. Albers et al., "Oral antithrombotic agents for the prevention of stroke in nonvalvular atrial fibrillation: a science advisory for healthcare professionals from the American HeartAssociation/American Stroke Association," Stroke, vol. 43, pp. 3442-3453, 2012.

[13] Atrial Fibrillation, "National Clinical Guidance for Management in Primary and Secondary care," http://www.nice.org .uk/nicemedia/live/10982/30055/30055.pdf, NICE 2006.

[14] Antithrombotics: Indications and Management. SIGN 129. A national clinical guidance, http://www.sign.ac.uk/pdf/SIGN129.pdf, 2012.

[15] J. Ansell, J. Hirsh, E. Hylek, A. Jacobson, M. Crowther, and G. Palareti, "Pharmacology and management of the vitamin $\mathrm{K}$ antagonists: American College of Chest Physicians EvidenceBased Clinical Practice Guidelines (8th edition)," Chest, vol. 133, supplement 6, pp. 160S-198S, 2008.

[16] A. J. Camm, G. Y. H. Lip, R. De Caterina et al., “2012 focused update of the ESC guidelines for the management of atrial fibrillation," European Heart Journal, vol. 33, pp. 2719-2747, 2012.

[17] F. W. Schofield, "A brief account of a disease in cattle simulating hemorrhagic septicemia due to feeding sweet clover," Canadian Veterinary Record, vol. 3, pp. 74-78, 1922.

[18] S. J. Connolly, M. D. Ezekowitz, S. Yusuf et al., "Dabigatran versus warfarin in patients with atrial fibrillation," The New England Journal of Medicine, vol. 361, no. 12, pp. 1139-1151, 2009.

[19] S. J. Connolly, M. D. Ezekowitz, S. Yusuf, P. A. Reilly, and L. Wallentin, "Newly identified events in the RE-LY trial," The New England Journal of Medicine, vol. 363, no. 19, pp. 1875-1876, 2010.

[20] K. Uchino and A. V. Hernandez, "Dabigatran association with higher risk of acute coronary events: meta-analysis of noninferiority randomized controlled trials," Archives of Internal Medicine, vol. 172, no. 5, pp. 397-402, 2012.

[21] P. Harper, L. Young, and E. Merriman, "Bleeding risk with dabigatran in the frail elderly," The New England Journal of Medicine, vol. 366, no. 9, pp. 864-866, 2012.

[22] M. R. Patel, K. W. Mahaffey, J. Garg et al., "Rivaroxaban versus warfarin in nonvalvular atrial fibrillation," The New England Journal of Medicine, vol. 365, no. 10, pp. 883-891, 2011.

[23] A. Rose, "Rivaroxaban versus warfarin in nonvalvular atrial fibrillation," The New England Journal of Medicine, vol. 365, no. 24, pp. 2333-2334, 2011.

[24] S. Schulman, S. Parpia, C. Stewart, L. Rudd-Scott, J. A. Julian, and M. Levine, "Warfarin dose assessment every 4 weeks versus every 12 weeks in patients with stable international normalized ratios a randomized trial," Annals of Internal Medicine, vol. 155, no. 10, pp. 653-659, 2011.

[25] M. Wieloch, A. Sjlander, V. Frykman, M. Rosenqvist, N. Eriksson, and P. J. Svensson, "Anticoagulation control in Sweden: reports of time in therapeutic range, major bleeding, and thrombo-embolic complications from the national quality registry AuriculA," European Heart Journal, vol. 32, no. 18, pp. 2282-2289, 2011.

[26] C. B. Granger, J. H. Alexander, and J. J. McMurray, "Apixaban versus warfarinin patients with atrial fibrillation," The New England Journal of Medicine, vol. 135, no. 11, pp. 981-992, 2011.

[27] V. S. Vassiliou, "Apixaban versus warfarin in atrial fibrillation," The New England Journal of Medicine, vol. 366, no. 1, p. 88, 2012.

[28] S. J. Conolly, J. Eikelboom, C. Joyner et al., "Apixaban in patients with atrial fibrillation," The New England Journal of Medicine, vol. 364, no. 9, pp. 806-817, 2011.

[29] R. D. Lopes, S. M. Al-Khatib, L. Wallentin et al., "Efficacy and safety of apixaban compared with warfarin according to patient risk of stroke and of bleeding in atrial fibrillation: a secondary analysis of a randomised controlled trial," The Lancet, vol. 380, pp. 1749-1758, 2012.

[30] V. S. Vassiliou and P. D. Flynn, "Apixaban in atrial fibrillation: does predicted risk matter?" The Lancet, vol. 380, pp. 1718-1720, 2012.

[31] J. B. Olesen, G. Y. H. Lip, J. Lindhardsen et al., "Risks of thromboembolism and bleeding with thromboprophylaxis in patients with atrial fibrillation: a net clinical benefit analysis using a "real world" nationwide cohort study," Thrombosis and Haemostasis, vol. 106, no. 4, pp. 739-749, 2011. 
[32] D. J. Scott, R. I. Dewar, C. J. Garratt et al., "RCPE UK consensus conference on 'approaching the comprehensive management of atrial fibrillation: evolution or revolution?"' The Journal Royal College of Physicians of Edinburgh, vol. 42, pp. 34-35, 2012.

[33] S. V. Shah and B. F. Gage, "Cost-effectiveness of dabigatran for stroke prophylaxis in atrial fibrillation," Circulation, vol. 123, no. 22, pp. 2562-2570, 2011. 


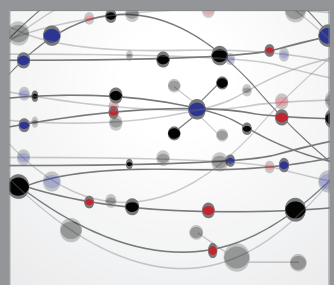

The Scientific World Journal
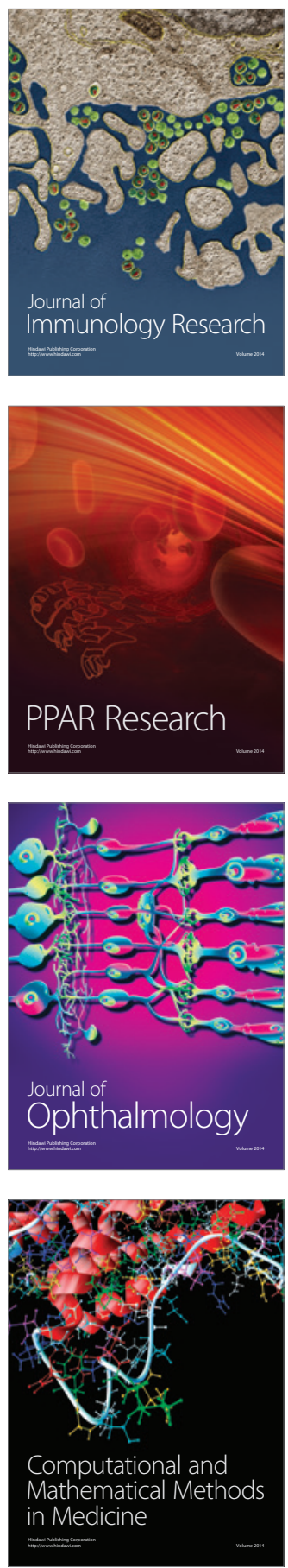

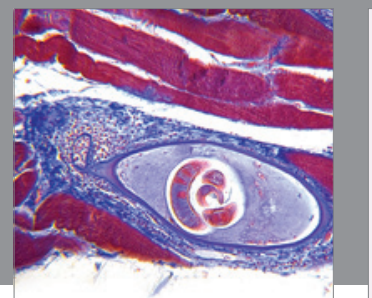

Gastroenterology

Research and Practice
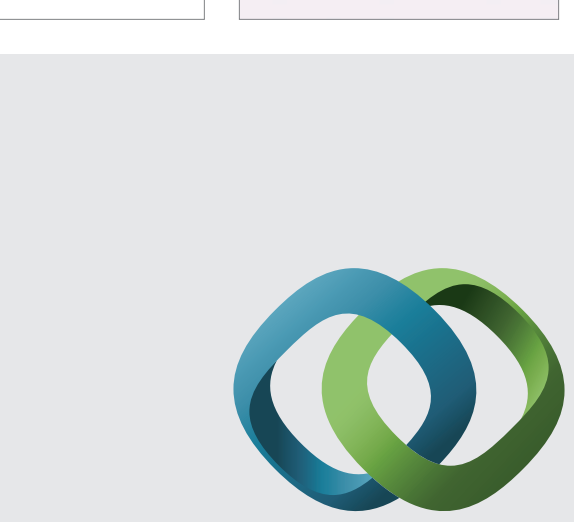

\section{Hindawi}

Submit your manuscripts at

http://www.hindawi.com
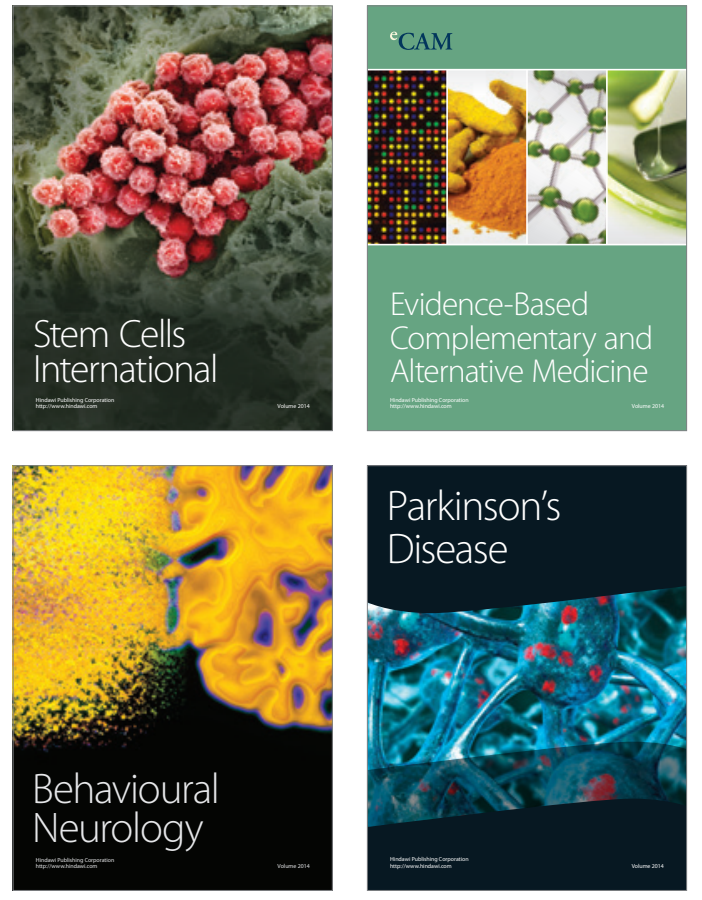
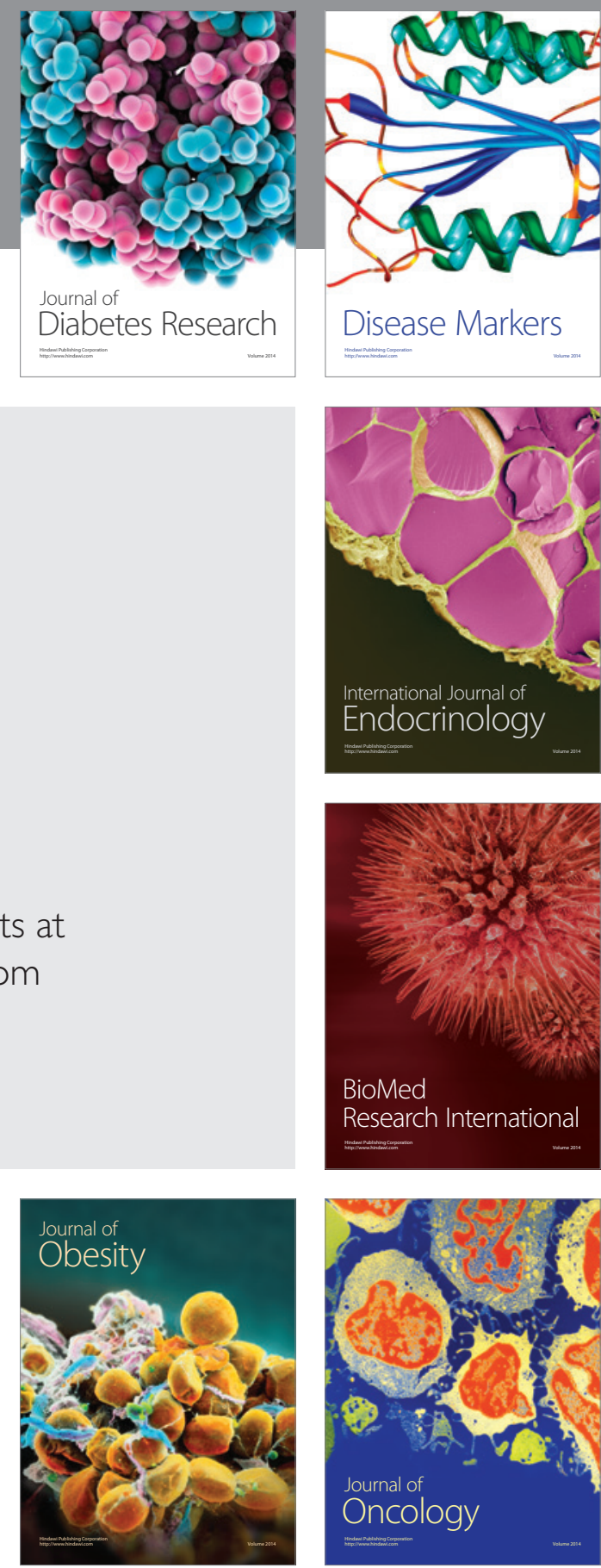

Disease Markers
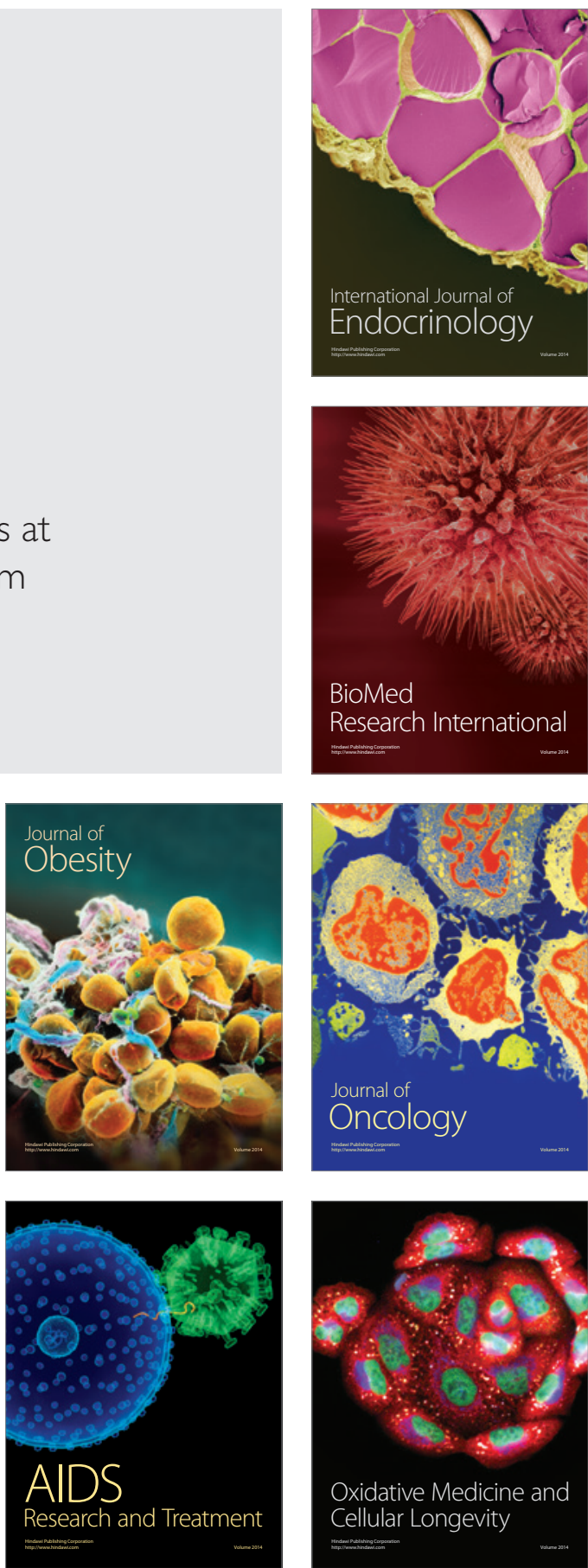